\title{
Optical Coherence Tomography Retinal and Optical Disc Imaging in Covid-19 Patients
}

\author{
Alyne Z. Simao*, Julia C. Andrade, Beatriz Harumi Yuda Nakagome, Gabriel C. Andrade, João Paulo \\ Muaccad Gama, Ronaldo Y. Sano, Niro Kasahara, Maria Auxiliadora M. Frazao \\ Department of Ophthalmology, Irmandade da Santa Casa de Misericórdia de Sao Paulo, Sao Paulo, Brazil
}

Abstract
Importance: To look for potential repercussions on the retinal microvasculature in patients with Covid-19
and thus contribute to a better understanding of organs target organs of this disease
Objective: To assess the retina of COVID-19 patients for possible microvascular damage.
Design: Uncontrolled observational cohort study.
Setting: Referral university hospital in Sao Paulo, Brazil.
Participants: 24 consecutive patients with laboratory diagnosis of COVID-19.
Exposure: Infection with SARS-CoV-2 and successive assessment of retina.
Main Outcome Measure: Qualitative interpretation of OCT retina and optical disc images.
Results: Most patients had no evident changes in the retina, nor the optical disc. Six eyes had nonspecific
changes with no clinical manifestation most possibly unrelated to SARS-CoV-2 infection.
Conclusion and Relevance: The patients with previous infection by SARS-CoV-2 evaluated in this study
did not present any images on OCT that can be associated to the virus.
Keywords: OCT retina, Covid-19, SARS-CoV-2.

\section{Introduction}

As of December, 2019, a new of a strain of coronavirus Severe Acute Respiratory Syndrome (SARS-CoV-2) emerged causing the pandemic of COVID-19. The disease affects mainly the respiratory system causing diffuse alveolar damage similar to injury caused by MERS-CoV and influenza virus. A unique feature of SARS-CoV-2 infection is vascular damage, with severe endothelial injury, widespread thrombosis, microangiopathy and angiogenesis [1]. The virus exploits the angiotensin-converting enzyme 2 (ACE2) receptor to gain entry inside the cells. Subsequent budding of the viral particles from the capillary endothelium and damage to the endothelial lining can favor viral access to the brain causing endothelial ruptures in cerebral capillaries accompanied by bleeding within the cerebral tissue [2]. This might explain the high incidence of thrombotic complications in intensive care unit patients with COVID-19 [3].

Corresponding Author: Alyne Z Simao, Rua Tomás Carvalhal, 970, Sao Paulo SP Brazil

Received date: November 19, 2020; Accepted date: November 26, 2020; Published date: November 27, 2020. DOI: https://doi.org/10.31546/2732-4516.1008 
Alyne Z. Simao et al.

COVID-19 infections are less frequent in children and they present with milder symptoms than adults. However, a few cases can be manifested as a hyperinflammatory syndrome with multiorgan involvement similar to Kawasaki disease shock syndrome [4].

The ocular manifestations of SARS-CoV-2 include keratoconjunctivitis and pseudomembranous hemorrhagic conjunctivitis [5,6]. Recently, hyper-reflective lesions at the retina as evidenced on optical coherence tomography (OCT), cotton wool spots and microhemorrhages were reported in eyes of a small cohort of COVID-19 patients [7]. In their study, however, some of the lesions resembled non-pathological blood vessels. Hence, the purpose of this study was to evaluate the retina of patients with laboratory diagnosis of COVID-19 using OCT and to correlated eventual findings with clinical ocular manifestations.

\section{Methods}

\section{Study design and setting}

This was an uncontrolled observational case series and included consecutive patients with laboratory diagnosis of SARS-CoV-2 infection presented to the Irmandade da Santa Casa de Sao Paulo Central Hospital, Sao Paulo, Brazil, from February, 2020 to June, 2020. The institution ethics committee approved the study protocol and the procedures followed the tenets of the Declaration of Helsinki and the Resolution 466/12, National Council of Health, Ministry of Health, Brazil. All participants signed the informed consent.

\section{Participants}

A search for all patients with diagnosis of COVID-19 was done using the electronic files and the medical charts were reviewed by three of us (AZS, JAC, BY). In order to be included in the study participants had to be older than 18 years of age, any gender, and positive diagnostic testing either by serologic assays to test for IgG, IgA, and IgM SARS-CoV-2 or quantitative reverse transcription polymerase chain reaction (RT-PCR) of the virus genoma. Patients with previous known retinal diseases such as diabetic retinopathy, posterior uveitis, or vitreo-retinal surgery were not included in order to avoid potential sources of bias. All patients who fulfilled the inclusion criteria were contacted and invited to participate in the study.

\section{Procedures}

After a brief medical interview - where demographic data was collected - participants underwent a brief ophthalmic exam including measurement of bestcorrected visual acuity on a Snellen chart, biomicroscopy with the slit-lamp (Topcon SL-D301, Topcon Corporation, Tokyo, Japan), indirect ophthalmoscopy (Heine Omega 500, Heine Optotechnik GmbH \& Co, Gilching, Germany), and optical coherence tomography (OCT) imaging.

\section{OCT images acquisition and interpretation}

Retina images of all participants were obtained with the DRI OCT Triton (Topcon Corporation, Tokyo, Japan) a posterior swept source OCT combined with multi modal fundus imaging device. It uses an optimized long wavelength scanning light $(1,050 \mathrm{~nm})$ with a scanning speed 100,000 A-Scans/second with 8 $\mu \mathrm{m}$ axial and $20 \mu \mathrm{m}$ lateral resolution in tissue. All scans were performed by the same experienced operator using standard technique. In brief, patients were positioned and instructed to fixate on an internal fixation light. The patient's first eye was aligned along the visual axis by a central fixation light; then device was brought into focus, with optimal alignment of the camera and even illumination of the entire image for the image acquisition. The quality and completeness of the data was verified immediately after. Images with poor signal strength, with segmentation errors, and/or with decentration of the scan were excluded and with the patient still positioned in front of the camera a new image taken again to get another of better quality. The 5 Line Cross scan, 3D Macula analysis, 3D Disc analysis, and the macula angiography scan $(4.5 \mathrm{~mm} \times$ $4.5 \mathrm{~mm}$ ) protocols were used to get images of the macula and optic disc areas.

OCT images of all patients were retrieved afterwards and assessed by two independent observers who interpreted every single image in a blinded fashion no clinical data was available except those displayed on the OCT printout. When divergent opinions between the observers aroused, a third expert would evaluate the image and all three would confirm a diagnosis in common agreement. 
Alyne Z. Simao et al.

\section{Statistical analysis}

For statistical analysis, each eligible eye was regarded as a unity of analysis. Data was tabulated on an Excel spread sheet (Microsoft Office, Microsoft Corp., Redmond, WA). For descriptive statistics categorical variables were presented as absolute frequencies and continuous variables were summarized using median (and range) or mean ( \pm standard deviation) when appropriate.

\section{Results}

Twenty-four patients (48 eyes) were included in the study. Detailed clinical and demographic features for everyone of the participants are depicted on table 1. Eleven patients were male and 13 female. Mean age was $38.5 \pm 14,06$ (range, 26 to 65). Five patients had some systemic comorbidity and 4 of them were on medication; two had previously undergone laser refractive surgery. Twenty-three patients presented typical symptoms of COVID-19 and included cough, fever, myalgia, dyspnea, and anosmia; only one patient was asymptomatic. Four patients had severe disease that necessitated hospitalization, one of them in the intensive care unit.
The median time between COVID-19 diagnosis and inclusion in the study was 53,5 days (range, 20 to 83 days) and all had recovered and were asymptomatic.

On ophthalmic examination, all 24 eyes had a bestcorrected visual acuity of $20 / 20$ on both eyes. Biomicroscopy showed no abnormalities in the conjunctiva, cornea, anterior chamber, iris and, lens in any of the eyes. Similarly, indirect ophthalmoscopy was within normal limits in both eyes of all patients.

Macula and optic disc OCT scans revealed no abnormality in most eyes (89.4\%). In 5 subjects (six eyes, nonspecific changes with no clinical manifestation were observed as follows: one eye presented a subtle elevation of the retinal pigment epithelium, possibly as the result of previous central serous chorioretinopathy; one eye with a micro-cyst $<1$ $\mathrm{mm}$ in the fovea; one eye presented a small hyperreflexive area in the superficial layers of the retina; one patient eye had a single cotton-wool spot (soft exudate) in the superior retina adjacent to the optic disc in one eye (figures 1 and 2) - the second eye had none; and one last patient presented non-specific low reflectivity region of the retina on both eyes.

Table 1: Detailed clinical and demographic features of the participants are depicted

\begin{tabular}{|c|c|c|}
\hline & $\mathbf{N}$ & $\%$ \\
\hline Gender & 24 & 100,0 \\
\hline Female & 13 & 54,2 \\
\hline Male & 11 & 45,8 \\
\hline Comorbidity & 24 & 100,0 \\
\hline No & 17 & 70,8 \\
\hline Yes & 7 & 29,2 \\
\hline \multicolumn{3}{|l|}{ Personal Records ${ }^{1,2}$} \\
\hline Asthma & 3 & 50,0 \\
\hline Allergic Rhinitis & 1 & 16,7 \\
\hline Fibromyalgia & 1 & 16,7 \\
\hline Arterial Hypertension, Cancer & 1 & 16,7 \\
\hline Childhood Toxoplasmosis & 1 & 16,7 \\
\hline No information & 1 & \\
\hline Use of medication & 24 & 100,0 \\
\hline No & 20 & 83,3 \\
\hline Yes & 4 & 16,7 \\
\hline Drug $^{3}$ & 4 & 100,0 \\
\hline Alenia & 1 & 25,0 \\
\hline PRISTIQ, LOSARTANA, TRULICITY & 1 & 25,0 \\
\hline REOVAR, VANISTO, CORTICOIDE INALATORIO & 1 & 25,0 \\
\hline
\end{tabular}


Alyne Z. Simao et al.

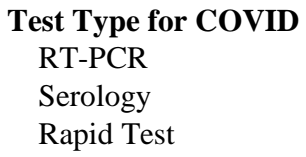

Symptom Onset Time (days)

asymptomatic - 0

From 2 to 5 days

From 6 to 10 days

From 11 to 15 days

Above 15 days

No information

Symptoms

Fever

No

Yes

Myalgia

No

Yes

Cough

No

Yes

50,0

Dyspnea

$24 \quad 100,0$

No

Yes

58,3

$\begin{array}{ll}14 & 58,3 \\ 10 & 41,7\end{array}$

Anosmia

$24 \quad 100,0$

No

Yes

Other Symptoms ${ }^{5}$

Headache

$15 \quad 78,9$

Arthralgia

Odinophagy

Retrorbital Pain

Dysgeusia

Nausea

Chest Pain

Diarrhea and sore throat

Mental confusion

No symptoms

$1 \quad 5,3$
Hospital Internment
No
Yes

Intensive Care Unit

No

Yes

100,0

95,8

4,2 
Treatment

No Treatment

Subcutaneous Enoxaparin
83,3

16,7

Alteration in slit lamp biomicroscopy ${ }^{5}$

Tarsal conjunctiva follicles

Blepharitis

Micropapils in tarsal conjunctiva

No modification

Results of optical coherence tomography

Physiological

Unilateral presence of elevation of the retinal pigment epithelium

Bulging of the RPE, ellipsoid zone and internal limiting membrane in the parafoveal region (both eyes)

Unilateral presence of two bulging points in the ellipsoid zone in a paradigmatic nasal region

Single Cotton-wool spot

Small hyper-reflexive area in the internal plexiform
57,1

42,9

14,3

1

17

24

19

100,0

79,2

4,2

4,2

4,2

4,2

4,2

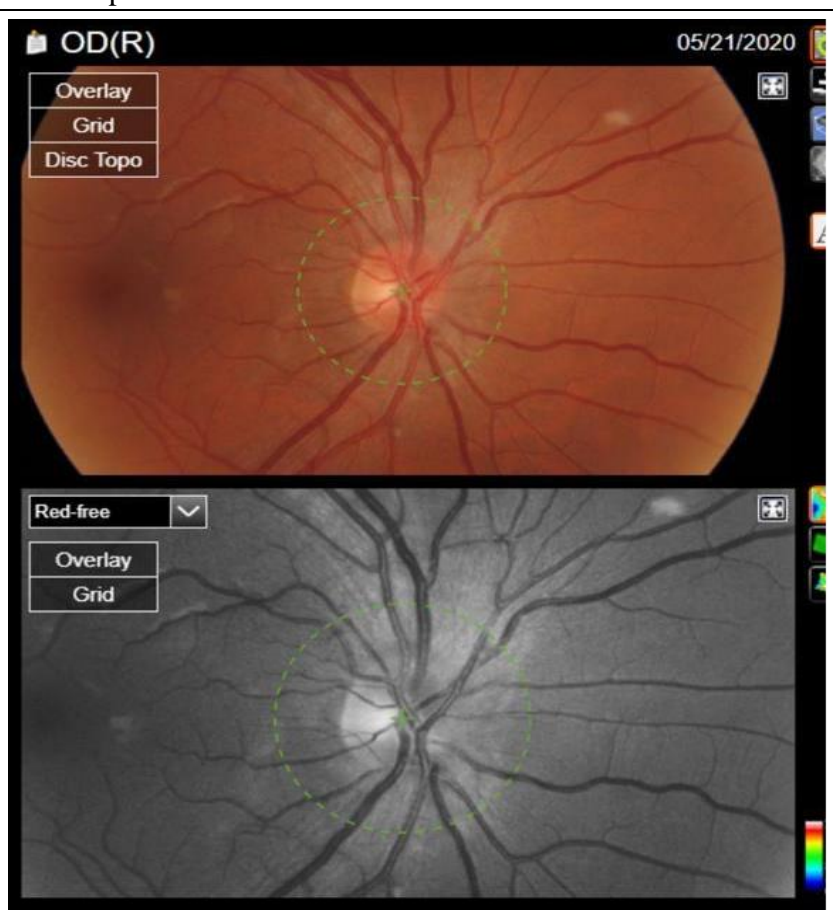

Figure 1: Single cotton-wool spot in the superior retina adjacent to the optic disc.
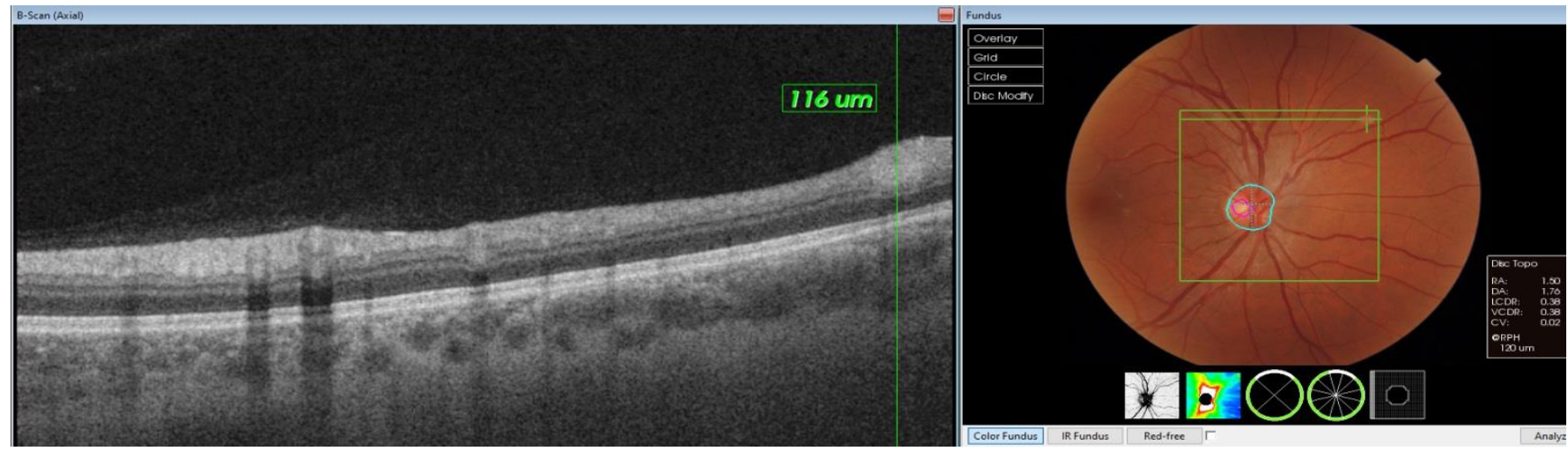

Figure 2: (Soft exudate with corresponding OCT 


\section{Discussion}

The study found no evidence of alteration attributable to Covid 19 in the retina, optic disc and retinal vasculature, constituting $89.47 \%$ of the eyes evaluated as absolutely normal, and the rest, no consistent changes that could be associated with previous infection by Covid-19 virus. Only a few patients had nonspecific changes with no clinical manifestation. We believe these findings were not related to the SARS-CoV-2.

The present study sought to find changes in the OCT examination in patients with a previous history of COVID 19, however, no changes were found as described in other studies, such as Marinho et. al. Indeed some concerns regarding the interpretation of the fundus and optical coherence tomography (OCT) findings have been seted up by the scientific community9. The study, of Vavvas et al. claims that these OCT findings bear significant resemblance to normal inner retinal vessels $[9,10]$ in terms of the morphology, reflectivity, location, and associated posterior shadowing, characteristic OCT features of blood vessels. Our study included a larger number of patients and very few had the so called hyperreflexive lesion.

Some methodological factors may have contributed to this divergence between the results, such as the device in which the exams of our study were carried out, the DRI OCT Triton (Topcon, Tokyo, Japan) that was also used in the study of Marinho et al, together with and XR Avanti SD-OCT (Optovue, Fremont, CA, USA). The population studied was another likely source of disagreement, once the study of the other group evaluated patients at a time closer to the acute phase of the disease. We evaluated patients who had recovered from COVID-19 and were past beyond active infection (20 to 83 days), whereas Marinho et al. evaluated patients shortly after COVID-19 symptom onset (11 to 33 days).

On the other hand, we adopted as an exclusion criterion all patients who did not present laboratory evidence of the disease, thus preventing symptomatic patients with another differential diagnosis from interfering in the data collection.

This study has some limitations. No patient was symptomatic when enrolled and most had recovered completely. Some of the transient changes in the retina of COVID-19 patients could only be diagnosed during the active phase of the disease and especially in those more severely sick with widespread vascular involvement. Nevertheless, the ophthalmic evaluation for a clinical study in this setting would not be a priority in patients struggling with their lives and probably unethical. Some patients were relatively young and had no risk factor for severe disease including hypertension, respiratory system disease, and cardiovascular disease. 8 Thus one would not expect to find any retinal injury related to the virus itself. OCT is a non-invasive technology developed to assess the retina and optic nerve. The circulation of the retina and choroid can be evaluated in details with the fluorescein angiography (FA) and contrast injection. We are unsure if early microvascular changes could be detected in COVID-19 patients with FA. Future studies including FA could be performed to acess possible findings.

There is one caveat to the generalisability of the results this study. The SARS-CoV-2 is in constant mutation. Different clinical manifestations can become rapidly evident as the evolutionary pattern of the virus changes towards new genetic variants. What may be true in COVID-19 patients from this sample might not be relevant to eventual new strains of coronavirus.

As it stands, the results of this study does not support the fact that COVID-19 patients are free from retinal involvement. There is evidence that SARS-CoV-2 causes endothelial injury and thrombosis in the lungs and cerebral tissue. The retina and the optic nerve as an extension of the brain are eventually prone to sustain the same damage. Besides that, more recently were found that Viral RNA of SARS-CoV-2 is detectable in the retina of COVID-19 patients 11 . Which further supports the thesis that there is an intraocular repercussion of the virus infection.

As a new disease, there still a lot to learn on the clinical features in different and peculiarities as to how the virus can affect human tissues in different countries and ethnic groups especially with new mutations in an ever evolving coronaviridae. We still believe that changes in the retina corresponding to retinal vascular distress can be identified in more acute 
Alyne Z. Simao et al.

phases, or in patients with more severe clinical conditions. Therefore further studies on the subject are still needed.

Funding: This research did not receive any specific grant from funding agencies in the public, commercial, or not-for-profit sectors.

Conflict of Interest: The authors declare no conflict of interest.

Patient Consent: Written informed consent was obtained from the patient for the publication.

\section{References}

1. Ackermann M, Verleden SE, Kuehnel M, et al. Pulmonary Vascular Endothelialitis, Thrombosis, and Angiogenesis in Covid-19. N Engl J Med. 2020;383:120-128.

2. Baig AM, Khaleeq A, Ali U, Syeda H. Evidence of the COVID-19 Virus Targeting the CNS: Tissue Distribution, Host-Virus Interaction, and Proposed Neurotropic Mechanisms. ACS Chem Neurosci. 2020;11:995-998.

3. Klok FA, Kruip MJHA, van der Meer NJM, et al. Incidence of thrombotic complications in critically ill ICU patients with COVID-19. Thromb Res. 2020;191:145-147.

4. Riphagen S, Gomez X, Gonzalez-Martinez C, et al. Hyperinflammatory shock in children during COVID-19 pandemic. Lancet. 2020;395:1607-1608.
5. Navel V, Chiambaretta F, Dutheil F. Haemorrhagic conjunctivitis with pseudomembranous related to SARS-CoV-2. Am J Ophthalmol Case Rep. 2020;19:100735.

6. Cheema M, Aghazadeh $\mathrm{H}$, Nazarali S, et al. Keratoconjunctivitis as the initial medical presentation of the novel coronavirus disease 2019 (COVID-19). Can J Ophthalmol. 2020;55:e125e129.

7. Marinho PM, Marcos AAA, Romano AC, et al. Retinal findings in patients with COVID-19. Lancet. 2020;395:1610.

8. Yang J, Zheng Y, Gou X, et al. Prevalence of comorbidities and its effects in patients infected with SARS-CoV-2: a systematic review and metaanalysis. Int J Infect Dis. 2020;94:91-95.

9. Vavvas DG, Sarraf D, Sadda SR. et al. Concerns about the interpretation of OCT and fundus findings in COVID-19 patients in recent Lancet publication. Eye (Lond). 2020;34:2153-2154.

10. Lee CS, Tyring AJ, Wu Y, et al. Generating retinal flow maps from structural optical coherence tomography with artificial intelligence. Sci Rep. 2019;9:5694.

11. Casagrande Maria, Fitzek Antonia, Püschel Klaus, et al. Detection of SARS-CoV-2 in Human Retinal Biopsies of Deceased COVID-19 Patients. Ocul Immunol Inflamm. 2020;28:721-725. 